\title{
Perception of shape-at-a-slant in the young infant
}

\author{
ROSE F. CARON \\ George Washington University, Washington, D.C. 20052 \\ ALBERT J. CARON and V. R. CARLSON \\ National Institute of Mental Health, Bethesda, Maryland 20014
}

and

LYNNE S. COBB

Purdue University, Lafayette, Indiana 47907

\begin{abstract}
In a recent study using recovery from habituation to index discrimination, the authors were unable to replicate Bower's shape-constancy findings. Since our habituation figures were steeply slanted, infants may have been unable to process their shape characteristics (real or proximal). To determine whether this was the case, two groups of 12-week-old infants were each habituated to one of the slanted figures used in the previous study and were then exposed to a new shape at the same slant. Significant recovery to the altered shape in both groups relative to a no-change control group indicated that the shape properties of the slanted objects had indeed been discriminated.
\end{abstract}

In a series of experiments using conditioning techniques, Bower $(1966 \mathrm{a}, 1966 \mathrm{~b})$ demonstrated that 8 -week-old babies can see the constant real shape of objects that have been rotated in depth so as to project differing shapes on the retina. Infants not only responded equivalently to a rectangle at varying backward slants but also discriminated it from a frontal trapezoid of identical retinal projection, thus leading Bower to conclude that early perception is attuned to objective rather than projective information.

Using recovery from habituation to index discrimination, Caron, Caron, and Carlson (1978) obtained only partial confirmation of Bower's (1966a, 1966b) results. Like Bower's subjects, their 12-week-old infants did not respond to equivalence of projective shape (i.e., they recovered to a frontal square following habituation to a large slanted trapezoid of identical projection) but, unlike Bower's subjects, they also overlooked constancy of true shape (recovering to the frontal square following habituation to its 60-deg orientation).

One possible explanation of these results is that infants may have been unable to process the shape characteristics (real or proximal) of the steeply slanted habituation figures and may simply have responded to change in slant or slant-related cues in both conditions. Failure to process the shape of slanted objects would be consistent with Bower's (1966b) proposal that young

The investigation was supported in part by Grant MH 25360 from the National Institute of Mental Health to the first author. The assistance of Camille D'Ascoli and Sandra Weiss is gratefully acknowledged. Requests for reprints should be sent to Rose F. Caron, Infant Research Laboratory, George Washington University, 10620 Georgia Avenue, Silver Spring, Maryland 20902. infants, because of "limited channel capacity," code only the single most salient attribute of a stimulus at any one time. Since strong depth cues were provided in the vicinity of the target in the Caron et al. (1978) situation, the most salient attribute may well have been slant.

This interpretation also gains credence from the fact there was no difference in magnitude of recovery between the 60-deg square and 60-deg trapezoid groups. If these figures had been perceived veridically, recovery should have been greater in the slanted trapezoid to frontal square condition (where there was a shift in real shape, size, and slant) than in the slanted square to frontal square condition (where there was a shift in slant alone). On the other hand, if only retinal information had been detected, the rank order of recovery should have been reversed (the rotated square group now involving a shift in retinal shape, size, and slant cues, and the trapezoid to square group a shift in proximal slant alone).

The purpose of the present study was to determine whether any aspect of shape, proximal or distal, had been discriminated in the Caron et al. (1978) experiment. The same figures used in that study were habituated at the same 60-deg slant, and only their shapes were altered at test, slant remaining constant. If the shape characteristics of such slanted objects are discriminable, significant recovery should occur to change in shape alone at test.

\section{METHOD}

\footnotetext{
Subjects

Three independent groups of 12-week-old healthy infants from middle-class suburban families served as subjects. There
} 
were 10 females and 8 males in each group (total $\mathrm{N}=54$ ). An additional 15 cases ( 5 males, 10 females) were eliminated because of poor state, and 14 more ( 4 males, 10 females) because of failure to meet various fixation criteria (see Stimuli and Procedure). The final sample ranged in age from 77 to 91 days, with the three groups having comparable means of 83.2, 84.0 , and 84.6 days.

\section{Apparatus}

The apparatus has been described in detail elsewhere (Caron et al., 1978). Briefly, the subject sat in a 45-deg inclined infant seat facing a similarly inclined $28 \times 30 \mathrm{~cm}$ (width $\times$ height) window that looked into either of two $28 \times 30 \times 32 \mathrm{~cm}$ (width $\mathrm{x}$ height $\mathrm{x}$ depth) compartments (one containing the habituation stimulus, the other the recovery stimulus). The white interior surfaces of the compartments contained black gridlines to provide maximum depth cues. The stimuli, positioned $45 \mathrm{~cm}$ from the infant's eyes, were attached to terminals of holder arms that projected into each compartment through a center aperture in the back cover. For present purposes, the terminals of the holder arms were all tilted 60 deg backward from the infant's line of regard. The box containing the two compartments could be moved along a grooved track so that either compartment overlaid the window, thereby permitting rapid change of stimuli. The luminance level in each compartment was $34.2 \mathrm{~cd} / \mathrm{m}^{2}$.

An aperture for videotaping the infant was located $6 \mathrm{~cm}$ to the left of the window at midlevel and, just above it, was a 1.4-W blue bulb that blinked at a 1-blink/sec rate during intertrial intervals to hold the child's attention. Fixation was scored from a TV monitor by a hand-held button coupled to a specialpurpose computer. The computer terminated each habituation trial upon the occurrence of a predetermined look-away criterion, and calculated on-line when the habituation criterion (see Stimuli and Procedure) had been met. When criterion was achieved, it delivered a tone to earphones worn by a second experimenter, who then manually moved the box to expose the second compartment for the ensuing test trials.

\section{Stimuli and Procedure}

There were three treatment groups, two experimental and one control, each of which saw the same standard figure at test: a $14.2-\mathrm{cm}$ planometric cardboard square tilted 60 deg backward. The groups differed only in terms of the stimuli presented for habituation. The control group was habituated to the identical 60-deg square. One experimental group received a stimulus equal in area to the square but discrepant in shape: a 60-deg backward trapezoid. Its upper edge measured $18.1 \mathrm{~cm}$, lower edge $10.3 \mathrm{~cm}$, and length, $14.2 \mathrm{~cm}$. The second experimental group saw a larger trapezoid, also at $60 \mathrm{deg}$, which in the Caron et al. (1978) experiment had served as the projective equivalent of the square in the frontoparallel plane. The ratio of its upper and lower edges (1.75) was the same as that of the small trapezoid (19.6 to $11.2 \mathrm{~cm}$; length $30.7 \mathrm{~cm}$ ). All three stimuli were bright red in hue and contained both an exterior and an interior white border.

Each trial cycle began with the activation of the blue light for a fixed $4.5 \mathrm{sec}$, following which a stimulus was exposed. Fixation was recorded from the child's initial orientation to the stimulus until the occurrence of a 2-sec look-away, whereupon the target lights were extinguished and the blue light reactivated for the next trial. The first trial (a warm-up) consisted of the presentation of a bright orange and black bullseye figure $(15.2 \mathrm{~cm}$ in diameter, $2.5 \mathrm{~cm}$ in depth). The next trial was the first habituation trial. Total fixation during the first three habituation trials combined constituted the base level for the computation of a relative habituation criterion, calculated as three successive trials totaling $50 \%$ or less of the initial level. An alternative absolute criterion (to prevent excessive exposure for those few subjects with low initial looking levels) consisted of three successive trials totaling $4.8 \mathrm{sec}$ or less. When criterion was achieved, the 60-deg slanted square was then exposed for two test trials to all three groups, following which the bullseye was again presented as a check on the infant's alertness.

Fixation, defined as the superimposition of the reflection of the target over the pupil of the infant's eye, was scored by observers who were blind to experimental condition. Reliability for this procedure had previously been established for the same observers as .93 (Caron et al., 1978). To ensure that all subjects had reasonably sufficient exposure to the habituation stimulus, infants were excluded from the sample whose combined total fixation over the first three habituation trials was less than $8 \mathrm{sec}$ and who habituated in less than seven trials.

\section{RESULTS}

\section{Prerecovery}

To determine the comparability of our treatment groups prior to recovery, each of the following scores was analyzed in terms of a 3 (stimulus group) by 2 (sex) ANOVA: (1) total fixation to the initial bullseye, (2) total fixation during the first three habituation trials, (3) number of trials to reach habituation criterion, and (4) total fixation on the last two habituation trials (H) (see Table 1).

There were no significant main or interaction effects involving sex. The only measure to yield a difference between stimulus groups was number of trials to habituation $[F(2,48)=4.23, \mathrm{p}<.05]$. Post hoc comparisons indicated that the group habituated on the large trapezoid required more trials to reach criterion than either the control group $(\mathrm{t}=2.79, \mathrm{p}<.01$, Dunnett's test) or those habituated on the small trapezoid $(q=2.99, p<.05$, Newman-Keuls). Inspection of the data suggests that this effect might have been due to the lower initial three-trial base of this group, thereby necessitating more trials to reach the criterial decrement

Table 1

Mean Habituation and Recovery Scores of the Experimental Groups

\begin{tabular}{lrcr}
\hline & \multicolumn{3}{c}{ Habituation Stimulus } \\
\cline { 2 - 4 } & LT & ST & CS \\
\hline \multirow{3}{*}{ Bullseye } & 14.2 & 15.4 & 10.6 \\
& \multicolumn{4}{c}{ Habituation } \\
First three trials & 11.7 & 16.2 & 13.0 \\
Number of trials & 10.7 & 8.2 & 7.4 \\
Last two trials (H) & 2.8 & 3.6 & 3.1 \\
& \multicolumn{4}{c}{ Recovery } \\
Two test trials (T) & 9.6 & 10.8 & 3.9 \\
Absolute increment (T-H) & 6.8 & 7.2 & .8 \\
Percent novelty fixation (T/T+H) & 72.5 & 66.0 & 49.8 \\
& \multicolumn{3}{c}{ Postrecovery } \\
Bullseye & 20.4 & 16.1 & 14.5 \\
\hline
\end{tabular}

Note-LT = 60-deg large trapezoid; $S T=60-\mathrm{deg}$ small trapezoid; $C S=60-d e g$ control square. Scores in seconds. 
of $50 \%$. Since stimuli identical to the large trapezoid and control figure were habituated in the Caron et al. (1978) study without the appearance of such a trial difference, the effect is likely a function of sampling rather than stimulus factors.

\section{Recovery}

The next three rows of Table 1 present the means of various recovery measures. The two relative scores, (1) the absolute increment in fixation from the last two habituations to the two test trials (T-H) and (2) total fixation on the test trials as a percentage of fixation on the test plus terminal habituation trials $(T /(T+H)$, were each submitted to a stimulus group by sex ANOVA. In each case, the overall effect of stimulus group, and no other effect, was significant [absolute increment, $F(2,48)=3.59, \mathrm{p}<.05$; percent test fixation, $F(2,48)=12.16, \mathrm{p}<.001$ ] . Post hoc comparisons revealed that on both measures the two experimental groups each differed reliably from the control but did not differ from each other $(\mathrm{p}<.05$ for both absolute comparisons, $\mathrm{p}<.01$ for both percentage comparisons, by Dunnett's tests).

\section{Postrecovery}

Mean total fixation on the final bullseye trial appears in the last row of Table 1. There were no significant effects here, indicating that the differences in recovery were not a function of differential attentiveness at test.

\section{DISCUSSION}

The results indicate that young infants are able to process the shape characteristics of slanted stimuli. While discrimination of size differences (real or projective) could have contributed to test performance in the large-trapezoid group, size cues could not have materially affected recovery in the small-trapezoid condition, given that this figure was identical in real size to the square and differed from it only minimally in projective size (4\%). The findings also help clarify the outcome of the earlier Caron et al. (1978) study. They suggest that the failure to obtain summative effects during recovery in that investigation was due to some factor other than the baby's inability to discriminate shape. In fact, shape appears to have been as salient as slant, since recovery to change in shape alone in the present experiment was as strong as that to alteration in slant alone in the previous study (66\% vs. $68 \%$, respectively). While we have no completely satisfactory explanation for the lack of summation, perhaps the presence of other stimulus attributes that remained constant between habituation and test (e.g., color and the white borders) so constrained the overall extent of recovery that no further effects of additivity could become manifest.

The present investigation and our earlier research, taken together, suggest that infants were processing shape and slant characteristics simultaneously during habituation. Our subjects could not have anticipated which of these features was to be varied at test, and yet, subsequent change in each alone yielded significant levels of recovery. This finding is at odds with Bower's (1966a, 1966b) contention that infants process only one attribute of an object at a time, an explanation invoked to account for the unresponsiveness of his subjects to change in slant when shape was simultaneously visible. Why variation in slant was not effective in Bower's (1966a) experiment is problematic, unless the remote distance employed $(2 \mathrm{~m})$ and the absence of depth cues in the immediate vicinity of the target reduced the salience of slant relative to shape. Another possibility is that Bower's use of a discrimination procedure with stimulus presence as the positive stimulus and stimulus absence as the negative stimulus emphasized the "thingness" of his conditioned target, and even for infants "things" may be more closely associated with shape than with slant. Whatever the case, it would seem inappropriate to regard the young infant as "limited" in processing capacity.

It should be noted, finally, that the present findings tell us that infants can respond to proximal variables correlated with shape and slant but not whether they transduce these sensory events into veridical percepts of real shapes-at-a-slant.

\section{REFERENCES}

Bower, T. G. R. Slant perception and shape constancy in infants. Science, 1966, 151, 832-834. (a)

Bower, T. G. R. The visual world of infants. Scientific American, 1966, 215, 80-92. (b)

Caron, A. J., Caron, R. F., \& Carlson, V. R. Do infants see objects or retinal images? Shape constancy revisited. Infant Behavior and Development, 1978, 1, 229-243.

(Received for publication January 3, 1979.) 